\title{
Acute pancreatitis in peritoneal dialysis and haemodialysis: risk, clinical course, outcome, and possible aetiology
}

\author{
M J Bruno, D J van Westerloo, W T van Dorp, W Dekker, J Ferwerda, G N J Tytgat, \\ N H Schut
}

\begin{abstract}
Background-It has been suggested that the incidence of acute pancreatitis in patients with end stage renal failure is increased.

Aims-To assess the risk of acute pancreatitis in patients on long term peritoneal dialysis and long term haemodialysis compared with the general population, to evaluate its clinical course and outcome, and to identify possible aetiological factors.
\end{abstract}

Patients-All patients who were maintained on long term peritoneal dialysis and/or haemodialysis (total dialysis time more than six weeks) from January 1989 to March 1998 in a large general hospital in The Netherlands.

Methods-Retrospective cohort study. Standardised ratios (as an approximate relative risk) between the incidence of acute pancreatitis in haemodialysis or peritoneal dialysis and the general population were calculated. Possible risk factors were identified. Patients with and without acute pancreatitis were compared.

Results-In 269 patients on haemodialysis (total of 614 person years), one patient developed an attack of acute pancreatitis. Patients on haemodialysis did not show an increased risk for acute pancreatitis compared with the general population (standardised ratio $11 ; 95 \%$ confidence interval (CI) 0.275 to 60.5 ). In 128 patients on peritoneal dialysis (total of 241 person years), seven patients had nine attacks of acute pancreatitis. Patients on peritoneal dialysis had a significantly and highly increased risk for acute pancreatitis (standardised ratio 249; 95\% CI 114 to 473). Mortality in this series of nine attacks was $11 \%$. No single aetiological risk factor could be identified.

Conclusions-The risk of acute pancreatitis in patients on long term peritoneal dialysis is significantly and highly increased compared with the general population. The underlying causal mechanisms remain to be elucidated.

(Gut 2000;46:385-389)

Keywords: acute pancreatitis; epidemiology; incidence; renal insufficiency; haemodialysis; peritoneal dialysis
In 1985 a case report was published on two patients with end stage renal failure who developed acute pancreatitis during continuous ambulatory peritoneal dialysis. ${ }^{1}$ This raised the question whether patients with end stage renal failure undergoing dialysis have an increased risk for acute pancreatitis. ${ }^{2}$ Subsequent reports seemed to confirm this assumption..$^{3-5}$ Some studies reported a significantly higher incidence of acute pancreatitis in patients on peritoneal dialysis compared with patients on haemodialysis. ${ }^{3}$ Others, however, did not confirm this observation. ${ }^{4}$

We have reviewed all the clinical data of patients undergoing long term peritoneal dialysis and/or haemodialysis in our dialysis centre over a nine year period. We assessed whether the incidence of acute pancreatitis in these patients is increased compared with the general population and calculated risk estimates. Furthermore, we evaluated its clinical course and outcome and attempted to identify possible aetiological factors.

\section{Patients and methods}

We retrospectively reviewed all medical data of patients undergoing chronic dialysis at the dialysis centre of the Kennemer Gasthuis, lokatie Elizabeth Gasthuis, Haarlem, The Netherlands, from January 1989 to March 1998. Chronic dialysis was defined as a total dialysis period longer than six weeks. Medical data that were reviewed included medical charts, laboratory reports, and reports from radiological investigations. An extensive database was created in which demographic data, data relating to dialysis (total dialysis time, type of dialysis, periods of dialysis, number of transplantations, cause of renal failure), and data relating to periods of pancreatitis (number of attacks, presenting symptoms, alcohol consumption, concomitant medication, history of peritonitis, amylase/lipase/calcium/triglyceride concentrations, peritoneal fluid cell counts and cultures, peritoneal fluid concentrations of amylase and lipase, findings on imaging investigations, total length of hospital stay, complications, and clinical course and outcome) were scored.

Acute pancreatitis was defined as a period with acute abdominal pain and discomfort with a more than threefold transient increase in serum amylase and/or lipase. To obtain "nor-

Abbreviations used in this paper: CT, computed tomography; US, ultrasound. 
mal" values of amylase and lipase activities in plasma and peritoneal fluid we randomly selected nine patients on long term haemodialysis and nine patients on long term peritoneal dialysis who had no history of acute pancreatitis. At the time of blood sampling none of these patients had symptoms or signs compatible with acute pancreatitis. In the random selection of patients on long term peritoneal dialysis without acute pancreatitis we also determined triglyceride and calcium concentrations. In order to identify possible aetiological risk factors for acute pancreatitis in peritoneal dialysis, we compared these "normal" values with concentrations in patients on peritoneal dialysis during their attack of acute pancreatitis.

\section{STATISTICS}

The incidence of acute pancreatitis in haemodialysis or peritoneal dialysis during the study period was calculated by dividing the number of cases by the total number of patients at risk. We calculated risk estimates such as the number of attacks of acute pancreatitis per person year and the number of patients per person year. The standardised ratio (incidence in haemodialysis or peritoneal dialysis/ incidence in general population) was calculated together with its $95 \%$ confidence interval (CI). The incidence of acute pancreatitis in The Netherlands from 1985 to 1995 was 15 per 100000 person years. ${ }^{6}$ This incidence rate was used to calculate the standardised ratios. Additionally, to test the firmness of these outcomes, "worst case" calculations were performed using the lowest and highest reported incidence of acute pancreatitis in northern Europe. The estimated incidence of acute pancreatitis in the general population for northern European countries is reported to be in the range from 5 to 73 per 100000 person years. ${ }^{7-11}$ For comparison of continuous variables, appropriate $t$ tests or non-parametric tests were used. For categorical data, the $\chi^{2}$ test for trend was used. Differences between groups were considered significant if the $\mathrm{p}$ value was less than 0.05 for a two tailed test.

\section{Results}

HAEMODIALYSIS

There were 269 patients (167 men and 102 women) undergoing long term haemodialysis. Their mean age was 64 years (range 22-87). The total duration of haemodialysis in these 269 patients was 614 person years (median duration 19 months, range 2-202). During this period one patient developed an attack of acute pancreatitis. The incidence of acute pancreatitis over the study period (9.3 years) was $0.4 \%$ $(1 / 269)$. There were $0.0016(1 / 614)$ attacks (and patients) of (with) acute pancreatitis per person year. The standardised ratio between the incidence of acute pancreatitis in haemodialysis and the general population was 11 and not significantly different from 1 (95\% CI 0.275 to $60.5)$. Using the lowest reported incidence in northern European countries (5 per 100000 person years) the standardised ratio was 54
(95\% CI 0.825 to 181 ), still showing no significantly increased risk.

The patient on haemodialysis who had an attack of pancreatitis finally underwent surgery because of an acute cholecystitis. During surgery a stone was found in the gall bladder neck. Peroperative cholangiography did not show stones in the common bile duct. As increases in amylase, lipase, and cholestatic liver tests were present before surgery and already dropping, pancreatitis in this patient was probably owing to a common bile duct stone or stones that had passed spontaneously. The clinical outcome was uneventful. The overall mortality rate of acute pancreatitis in patients on haemodialysis was $0 \%$.

\section{PERITONEAL DIALYSIS}

There were 128 patients (84 men and 44 women) undergoing long term peritoneal dialysis. Their median age was 59 years (range 20-81). The total duration of peritoneal dialysis in these 128 patients was 241 person years (median duration 17 months, range 2-92). During this period seven patients had nine attacks of acute pancreatitis. The incidence of acute pancreatitis in peritoneal dialysis during the study period ( 9 years and 3 months) was $5.4 \%$ (7/128). There were 0.037 (9/241) attacks of acute pancreatitis per person year and $0.029(7 / 241)$ patients per person year. The standardised ratio between the incidence of acute pancreatitis in peritoneal dialysis and the incidence in The Netherlands was 249 , and highly and significantly different from 1 (95\% CI 114 to 473). Using the highest reported incidence in northern European countries (73 per 100000 patient years) the standardised ratio is 51 (95\% CI 23 to 97), still showing a significantly increased risk. The incidence of acute pancreatitis between long term haemodialysis and peritoneal dialysis was also significantly different $(\mathrm{p}<0.001)$.

\section{Presentation and patient history}

Six patients had one attack of acute pancreatitis. One patient had three attacks. In between these attacks this patient had no abdominal complaints and serum amylase and lipase concentrations returned to normal. Median time on peritoneal dialysis until the attack of acute pancreatitis was 16 months with a wide range of 3-48 months. Four of seven patients had been maintained only on peritoneal dialysis and had never undergone haemodialysis. Three of seven patients had earlier episodes of haemodialysis (durations of 1, 20, and 41 months). Two of seven patients had a history of renal transplantation with rejection. None of the patients used medication known to be causally related to acute pancreatitis. ${ }^{12}$ None of the patients had a history of alcohol abuse. Only two of seven patients had a history of peritonitis.

\section{Laboratory dato}

Table 1 lists the (peak) serum amylase, (peak) serum lipase, amylase, and lipase concentrations in peritoneal fluid for each individual patient together with the results of both imaging 
Table 1 Laboratory parameters and imaging results and a selection of outcome parameters listed for each patient who developed acute pancreatitis while on peritoneal dialysis (PD)

\begin{tabular}{|c|c|c|c|c|c|c|c|c|c|c|c|}
\hline Patient no & $\begin{array}{l}\text { Amylase at } \\
\text { admission } \\
(U / l)\end{array}$ & $\begin{array}{l}\text { Peak } \\
\text { amylase } \\
\text { (U/l) }\end{array}$ & $\begin{array}{l}\text { Lipase at } \\
\text { admission } \\
\text { (Ull) }\end{array}$ & $\begin{array}{l}\text { Peak } \\
\text { lipase } \\
\text { (U/l) }\end{array}$ & $\begin{array}{l}\text { Amylase in } \\
\text { PD fluid } \\
\text { (U/l) }\end{array}$ & $\begin{array}{l}\text { Lipase in } \\
\text { PD fluid } \\
\text { (U/l) }\end{array}$ & Ultrasound ${ }^{\star}$ & $C T \operatorname{scan}^{\star}$ & $\begin{array}{l}\text { Necrotising } \\
\text { pancreatitis }\end{array}$ & Pseudocyst & Death \\
\hline 1 & 100 & 104 & 13100 & 13100 & 30 & 361 & Positive & Positive & Yes & Yes & Yes \\
\hline 2 & 192 & 406 & 849 & 2144 & 30 & 15 & Positive & Negative & No & No & No \\
\hline 3 & 340 & 451 & 1978 & 3818 & 30 & 1 & Positive & Positive & No & No & No \\
\hline 4 -attack 1 & 251 & 659 & 3070 & 4510 & 130 & 144 & $\begin{array}{l}\text { Not } \\
\text { visualised }\end{array}$ & Positive & No & No & No \\
\hline 4 -attack 2 & 323 & 1070 & 2310 & 14000 & 33 & 46 & $\begin{array}{l}\text { Not } \\
\text { performed }\end{array}$ & Negative & No & No & No \\
\hline 4 -attack 3 & 589 & 1070 & 5640 & 6730 & 616 & 1336 & $\begin{array}{l}\text { Not } \\
\text { performed }\end{array}$ & Negative & No & No & No \\
\hline 5 & 1150 & 1150 & 7780 & 7780 & 62 & 561 & $\begin{array}{l}\text { Not } \\
\text { visualised }\end{array}$ & Positive & No & Yes & No \\
\hline 6 & 1339 & 1339 & 8620 & 8620 & 1229 & 21950 & $\begin{array}{l}\text { Not } \\
\text { visualised }\end{array}$ & Positive & Yes & No & No \\
\hline 7 & 482 & 482 & 5700 & 5700 & $\begin{array}{l}\text { Not } \\
\text { available }\end{array}$ & $\begin{array}{l}\text { Not } \\
\text { available }\end{array}$ & Negative & Positive & No & Yes & No \\
\hline
\end{tabular}

₹Initial examination.

techniques and a selection of outcome parameters. Table 2 lists the mean/median outcomes of (peak) serum amylase, (peak) serum lipase, amylase, and lipase concentrations in peritoneal fluid, triglyceride concentration, and calcium concentration in patients on long term peritoneal dialysis who suffered from an attack of acute pancreatitis and the random selection of patients on peritoneal dialysis who did not. Serum triglyceride and calcium concentrations did not differ significantly between both groups. In all of the randomly selected patients on peritoneal dialysis without acute pancreatitis, peritoneal lipase and amylase concentrations were below $10 \mathrm{U} / 1$ and $30 \mathrm{U} / 1$, respectively. Peritoneal lipase concentrations rose above $10 \mathrm{U} / 1$ during seven of the total of nine attacks of acute pancreatitis. Peritoneal amylase concentrations rose above $30 \mathrm{U} / 1$ during five attacks. Five

Table 2 Laboratory outcomes; comparison between patients on peritoneal dialysis (PD) with and without pancreatitis

\begin{tabular}{lll}
\hline & PD without pancreatitis & PD with pancreatitis \\
\hline Serum amylase $(\mathrm{U} / \mathrm{l})^{\star}$ & $81(28), 47-131$ & $530(433), 100-1339$ \\
Peak serum amylase $(\mathrm{U} / \mathrm{l})^{\star}$ & - & $748(421), 104-1339$ \\
Serum lipase $(\mathrm{U} / \mathrm{l})^{\star}$ & $202(139), 81-460$ & $5450(3919), 849-13100$ \\
Peak serum lipase $(\mathrm{U} / \mathrm{l})^{\star}$ & - & $7378(4028), 2144-14000$ \\
Amylase peritoneal fluid $(\mathrm{U} / \mathrm{l}) \dagger$ & $<30$ & $48,30-1229$ \\
Lipase peritoneal fluid $(\mathrm{U} / \mathrm{l}) \dagger$ & $<10$ & $253,1-21950$ \\
Serum triglyceride $(\mathrm{mmol} / \mathrm{l}) \ddagger$ & $3.6(2.0), 1.5-5.4$ & $2.6,1.1-9.3$ \\
Serum calcium $(\mathrm{mmol} / \mathrm{l}) \ddagger$ & $2.60(0.14), 2.40-2.84$ & $2.42(0.28), 2.20-3.06$ \\
\hline
\end{tabular}

${ }^{\star}$ Mean (SD), range.

tMedian, range.

$\neq \mathrm{p}>0.05$.

Table 3 Presence of known risk factors and factors that have been postulated to be associated with acute pancreatitis in peritoneal dialysis (PD)

\begin{tabular}{llllllllll}
\hline & $H C$ & $H T$ & $C H$ & $A L$ & $T H$ & $M E$ & $H P$ & $C P$ & Total $^{*}$ \\
\hline Patient 1 & + & + & - & - & - & - & + & + & 4 \\
Patient 2 & - & - & - & - & - & - & - & - & 0 \\
Patient 3 & - & + & - & - & - & - & - & - & 1 \\
Patient 4 & & & & & & & & & \\
Attack 1 & - & NA & - & - & - & - & - & - & 0 \\
Attack 2 & + & - & - & - & - & - & - & - & 1 \\
Attack 3 & + & - & - & - & - & - & - & - & 1 \\
Patient 5 & - & + & - & - & + & - & - & - & 2 \\
Patient 6 & - & - & + & - & - & - & + & - & 2 \\
Patient 7 & - & + & - & - & + & + & - & + & 4 \\
Totalt & 3 & 4 & 1 & 0 & 2 & 1 & 2 & 2 &
\end{tabular}

*Total number of factors per patient.

†Times that risk factor was present during a total of nine attacks of acute pancreatitis.

+, yes; -, no; NA, not available; HC, hypercalcaemia; HT, hypertriglyceridaemia; $\mathrm{CH}$, choledocholithiasis; AL, alcohol; TH, transplantation history; ME, medication; HP, history of peritonitis; $\mathrm{CP}$, concomitant peritonitis. patients had negative peritoneal fluid culture. Four of these patients had normal peritoneal fluid cell counts while one patient had a cell count of 1100 cells $/ \mathrm{mm}$. In two patients peritoneal fluid cultures were positive (Gram positive cocci and Gram negative rods with yeast). These patients had peritoneal fluid cell counts of 27 and 2700 cells $/ \mathrm{mm}$, respectively.

\section{Imaging investigations}

Ultrasound (US) showed a normal pancreas in two patients, pancreatitis in three patients, and could not visualise the pancreas in the remaining two patients (table 1). Computed tomography (CT) showed signs of pancreatitis in six patients. The patient with negative CT findings showed signs of pancreatitis during US. Most probably, this is explained by the fact that the CT scan was performed a few days after the US examination at which time the swelling of the pancreas had already disappeared. In the patient who had three attacks of pancreatitis, CT showed oedema of the pancreatic tail area only during the first attack. CT was normal during the subsequent two attacks. Endoscopic retrograde cholangiopancreatography (ERCP) was performed in three patients. In one patient choledocholithiasis was found and the calculi were removed; in one patient it was normal, and in one patient cannulation failed.

Clinical course, outcome, and possible aetiology The median number of days spent in the hospital was 37 (range 16-113). Five patients had oedematous pancreatitis while in two patients necrotising pancreatitis developed. Three patients developed pseudocysts. One patient died 113 days after hospital admission. This patient developed a large pseudocyst that was drained transgastrically by means of endoscopy. Because of a persisting bleed from the puncture aperture she underwent a laparotomy at which time areas with extensive pancreatic necrosis were found. Debridement and a cystgastrostomy were performed. This patient finally died because of respiratory insufficiency and multiple organ failure. All the other patients recovered uneventfully. The overall mortality rate of acute pancreatitis in peritoneal dialysis calculated per patient was $14 \%(1 / 7)$ and counted per attack $11 \%(1 / 9)$. 
Table 3 lists known risk factors and factors that have been postulated to be associated with acute pancreatitis in peritoneal dialysis (see Discussion). From this table it becomes clear that there is no single aetiological factor that can be identified in our patients.

\section{Discussion}

At postmortem examinations pancreatic abnormalities are reported in up to $60 \%$ of patients who were maintained on long term haemodialysis. ${ }^{13-15}$ Histological changes were found to be diffusely present throughout the pancreas and included duct ectasia, periductal fibrosis, ductular proliferation, acinar ductular metaplasia, inspissated secretions, atrophy, thickening and fibrosis of arterioles, haemosiderin deposits, calcifications, amyloidosis, hyalinisation, cystic changes, necrosis of peripancreatic fat, and abscess formation. This high prevalence of pancreatic pathology after long term haemodialysis does not seem to match the incidence of clinically observed pancreatic disease (for example, acute/chronic pancreatitis and exocrine pancreatic insufficiency). However, the true incidence and prevalence of exocrine pancreatic insufficiency in long term haemodialysis or peritoneal dialysis is unknown. Autopsy data regarding patients who were maintained on long term peritoneal dialysis are not available.

In patients with renal insufficiency concentrations of gastrointestinal hormones such as cholecystokinin, serum gastric inhibitory polypeptide, and glucagon are significantly increased in relation to the degree of renal impairment. ${ }^{16}$ During long term haemodialysis these increased hormone concentrations do not return to normal. This increase in hormone concentrations causes hypersecretion of pancreatic enzymes, predominantly trypsin. ${ }^{17}$ The continued stimulation of the exocrine pancreas by cholecystokinin may account for the morphological changes that are observed in these patients. This may eventually lead to impaired pancreatic function. Therefore, in the differential diagnosis of the wasting syndrome that is sometimes seen in patients on chronic dialysis, exocrine pancreatic insufficiency with energy losses owing to steatorrhoea should be considered. ${ }^{18}$

In the absence of acute pancreatitis, plasma concentrations of amylase and lipase are frequently increased in uraemic patients and patients on chronic dialysis, both haemodialysis and peritoneal dialysis. ${ }^{19}$ However, serum amylase activity greater than a threefold increase, together with acute onset abdominal pain is suggestive of acute pancreatitis. If there is doubt, determination of amylase isoenzymes, serum lipase concentrations, and lipase and amylase concentrations in the peritoneal fluid are useful to help distinguish a pancreatic from a non-pancreatic aetiology. ${ }^{20}$ Whenever a patient on long term peritoneal dialysis presents with acute onset abdominal pain, even in the presence of increased concentrations of amylase and/or lipase, (concomitant) bacterial peritonitis should be excluded by peritoneal fluid cell counts and cultures. If dialysate amy- lase concentrations are at least $100 \mathrm{IU} / 1$, acute pancreatitis is highly probable. In our patients, taking into consideration the clinical picture, the results of laboratory investigations (together with resolution to normal concentrations after the attacks), and the outcome of the imaging investigations, the diagnosis acute pancreatitis seems without doubt.

The 10 year incidence of acute pancreatitis in patients with end stage renal disease (without further specification as to whether or not and what type of renal replacement therapy was started) is reported to be around $2.5 \% .^{321}$ In our series the 10 year incidence (recalculated from 9.25 years) was $0.4 \%$ for haemodialysis and $5.8 \%$ for peritoneal dialysis. The incidence per 100 person years is reported to be in the range from 0.63 to 1.41 in long term haemodialysis and from 0.46 to 4.3 in long term peritoneal dialysis. ${ }^{3521}$ We found incidence rates of 0.16 and 4.3 , respectively. The remarkably lower incidence rate of acute pancreatitis in peritoneal dialysis $(0.46$ per 100 person years) reported by Pannekeet et al may be owing to recall bias. In this study, cases of acute pancreatitis during peritoneal dialysis were identified by means of interviews with nephrologists in dialysis centres. It is not unlikely that physicians who were interviewed were more likely to recall the more severe cases. This seems also to be reflected by the extremely high mortality rate of $58 \%$ in this series. Other groups also observed a high mortality rate but, as stated by the authors, less severe cases of acute pancreatitis may easily have been missed because symptoms of acute pancreatitis were often similar to those of dialysis associated peritonitis. $^{2}$ We observed a mortality rate of $11 \%$ of acute pancreatitis in long term peritoneal dialysis that does not differ from the reported overall (general population) mortality rate of acute pancreatitis in controlled clinical trials. In agreement with previous studies we noted that acute pancreatitis occurred more frequently in peritoneal dialysis than in haemodialysis. $^{2321}$ In fact, in our series we observed no increased risk for acute pancreatitis for patients on haemodialysis compared with the general population. The $95 \%$ confidence interval, however, is very broad. One additional patient on haemodialysis with acute pancreatitis would yield a rate ratio of 21.7 (95\% CI 2.63 to 78.4 ), indicating a significantly higher risk of acute pancreatitis in haemodialysis.

Of great interest and significant importance is the pathophysiological mechanism that may be responsible for the highly increased risk of acute pancreatitis in long term peritoneal dialysis. At first our results suggested that it is associated with the act of peritoneal dialysis and not with renal insufficiency as such. However, as stated above, the sample size may have been too small to detect a statistically significant difference between the general population and patients on haemodialysis. Others did find a higher incidence of acute pancreatitis in haemodialysis compared with the general population. An increased incidence of acute pancreatitis after renal transplantation is also 
reported but may be related to the surgery and the use of immunosuppressive agents. The risk of developing pancreatitis in long term peritoneal dialysis may be explained by a summation of separate risk factors: general population risk factors, risk factors related to renal insufficiency (uraemia, secondary hyperparathyroidism with hypercalcaemia, hypertriglyceridaemia, use of drugs), and as yet unidentified risk factors related to the act of peritoneal dialysis. With respect to the latter one could speculate that chronic infusion of a large amount of fluid with a non-physiological composition under non-physiological high intraabdominal pressure renders the pancreas more susceptible to parenchymal damage. Impairment of microvascularisation and hypoxaemia may induce premature activation of proteolytic enzymes, thereby provoking acute pancreatitis. The increase in intra-abdominal pressure during long term peritoneal dialysis is considerable and is known to cause complications such as herniation, prolapse of the uterus or rectum, and rarely hydrothorax. The composition of the dialysate may also (indirectly) influence factors known to be related to acute pancreatitis such as induction or aggravation of hyperglycaemia because of high glucose concentrations leading to hyperlipidaemia. Moreover, increased concentrations of pancreatic stimulating hormones together with the high prevalence of morphological changes may render the pancreas more susceptible to stimuli that potentially provoke acute pancreatitis.

More than one "known" or proposed risk factor was present in four of seven patients (table 2), the most prevalent one being hypertriglyceridaemia in four of seven patients. However, serum triglyceride concentrations below $5.64 \mathrm{mmol} / 1$ are less likely to be associated with pancreatitis and this level was exceeded in one patient only $(9.3 \mathrm{mmol} / \mathrm{l}) .^{22}$ Overall there was no statistically significant difference between serum triglyceride concentrations in patients on long term peritoneal dialysis with and without acute pancreatitis. The cumulative effect of increased triglyceride concentrations as well as increased calcium concentrations (possibly masked by low albumin concentrations) is not known. The cumulative duration of chronic dialysis did not seem to be a determining factor in our study. This is in agreement with Padilla et al who also observed a wide range in the time interval between the start of treatment and the onset of acute pancreatitis for both haemodialysis and peritoneal dialysis. ${ }^{21}$ Others suggested that a history of recurrent or concomitant episode(s) of peritonitis might be a risk factor for acute pancreatitis by leakage of infected peritoneal fluid into the lesser sac or haematogeneous spread to the peripancreatic region. Based on our results we cannot refute or confirm this hypothesis.

From the results of our series and previous studies it becomes clear that there is no single aetiological factor that accounts for the increased incidence of acute pancreatitis in peritoneal dialysis. Its pathophysiological mechanism seems multifactorial. There seems to be a stepwise risk increase for acute pancreatitis from the general population to patients with renal insufficiency (who may be maintained on haemodialysis) and finally to patients on long term peritoneal dialysis. The latter step, according to the results of this study, adds greatly to the overall risk. The causal mechanism by which long term peritoneal dialysis increases the risk of acute pancreatitis remains as yet unknown.

1 Pitrone F, Pellegrino E, Mileto G, et al. May pancreatitis represent a CAPD complication? Report of two cases with a rapidly evolution to death. Int f Artif Organs 1985;8:235.

2 Caruana RJ, Wolfman NT, Karstaedt N, et al. Pancreatitis: an important cause of abdominal symptoms in patients on peritoneal dialysis. Am f Kidney Dis 1986;7:135-40.

3 Rutsky EA, Robards M, Van Dyke JA, et al. Acute pancreatitis in patients with end-stage renal disease without transplantation. Arch Intern Med 1986;146:1741-5.

4 Gupta A, Yuan ZY, Balaskas EV, et al. CAPD and pancreatitis: no connection. Perit Dial Int 1992;12:309-16.

pancreatitis: no connection. Perit Dial Int 1992;12:309-16.
5 Pannekeet MM, Krediet RT, Boeschoten EW, et al. Acute pancreatitis during CAPD in The Netherlands. Nephrol Dial Transplant 1993;8:1376-81.

6 Eland IA, Sturkenboom MJCM, Wilson JHP, et al. Incidence and mortality of acute pancreatitis in the Netherlands between 1985 and 1995. Eur F Gastroenterol Hepatol 1998;10:A87.

7 Halvorsen FA, Ritland S. Acute pancreatitis in Buskerud County, Norway. Incidence and etiology. Scand $\mathcal{F}$ Gastroenterol 1996;31:411-14.

8 Worning H. Acute pancreatitis in Denmark. Ugeskr Laeger 1994;156:2086-9.

9 Katschinski BD, Giggs JA, Bourke JB. Incidence and geographical distribution of acute pancreatitis in Nottingham 1969 to 1983. Z Gastroenterol 1990;28:183-7.

10 Trapnell JE, Duncan EH. Patterns of incidence in acute pancreatitis. BMF 1975;ii: $179-83$.

11 Jaakkola $M$, Nordback I. Pancreatitis in Finland between 1970 and 1989. Gut 1993;34:1255-60.

12 Runzi M, Layer P. Drug-associated pancreatitis: facts and Runzi M, Layer P. Drug-associated
fiction. Pancreas 1996;13:100-9.

13 Baggenstoss AH. The pancreas in uremia: a histopathological study. Am $\mathcal{F}$ Pathol 1948;24:1003-11.

14 Avram MM. High prevalence of pancreatic disease in chronic renal failure. Nephron 1977;18:68-71.

15 Vaziri ND, Dure-Smith B, Miller R, et al. Pancreatic pathology in chronic dialysis patients-an autopsy study of 78 cases. Nephron 1987;46:347-9.

16 Owyang C, Miller LJ, DiMagno EP, et al. Gastrointestinal hormone profile in renal insufficiency. Mayo Clin Proc 1979;54:769-73.

17 Owyang C, Miller LJ, DiMagno EP, et al. Pancreatic exocrine function in severe human chronic renal failure. Gut 1982;23:357-61

18 Sachs EF, Hurwitz FJ, Bloch HM, et al. Pancreatic exocrine hypofunction in the wasting syndrome of end-stage renal disease. Am $\mathcal{F}$ Gastroenterol 1983;78:170-3.

19 Royse VL, Jensen DM, Corwin HL. Pancreatic enzymes in Royse VL, Jensen DM, Corwin HL. Pancreatic enzymes
chronic renal failure. Arch Intern Med 1987;147:537-9.

chronic renal failure. Arch Intern Med 1987;147:537-9.
20 Jensen DM, Royse VL, Bonello JN, et al. Use of amylase isoenzymes in laboratory evaluation of hyperamylasemia. isoenzymes in laboratory evalu
Dig Dis Sci 1987;32:561-8.

21 Padilla B, Pollak VE, Pesce A, et al. Pancreatitis in patients with end-stage renal disease. Medicine (Baltimore) 1994;73: 8-20

22 Toskes PP. Recurrent acute pancreatitis. Hosp Pract 1985;20:85-92. 